\title{
Multiple Feature Vectors Based Fault Classification for WSN Integrated Bearing of Rolling Mill
}

\author{
Bo Qin $\left(\mathbb{D},{ }^{1}\right.$ Luyang Zhang $\mathbb{D}^{1},{ }^{1}$ Heng Yin $\mathbb{D}^{1},{ }^{1}$ and Yan Qin $\mathbb{D}^{2}$ \\ ${ }^{1}$ School of Mechanical Engineering, Inner Mongolia University of Science and Technology, Baotou 014010, China \\ ${ }^{2}$ College of Control Science and Engineering, Zhejiang University, Hangzhou 310027, China \\ Correspondence should be addressed to Yan Qin; neuqinyan@163.com
}

Received 21 September 2017; Accepted 1 February 2018; Published 1 April 2018

Academic Editor: Yongji Wang

Copyright (c) 2018 Bo Qin et al. This is an open access article distributed under the Creative Commons Attribution License, which permits unrestricted use, distribution, and reproduction in any medium, provided the original work is properly cited.

\begin{abstract}
For rolling mill machines, the operation status of bearing has a close relationship with process safety and production effectiveness. Therefore, reliable fault diagnosis and classification are indispensable. Traditional methods always characterize fault feature using a single fault vector, which may fail to reveal whole fault influences caused by complex process disturbances. Besides, it may also lead to poor fault classification accuracy. To solve the above-mentioned problems, a fault extraction method is put forward to extract multiple feature vectors and then a classification model is developed. First, to collect sufficient data, a data acquisition system based on wireless sensor network is constructed to replace the traditional wired system which may bring dangers during production. Second, the measured signal is filtered by a morphological average filtering algorithm to remove process noise and then the empirical mode decomposition method is applied to extract the intrinsic mode function (IMF) which contains the fault information. On the basis of the IMFs, a time domain index (energy) and a frequency index (singular values) are proposed through Hilbert envelope analysis. From the above analysis, the energy index and the singular value matrix are used for fault classification modeling based on the enhanced extreme learning machine (ELM), which is optimized by the bat algorithm to adjust the input weights and threshold of hidden layer node. In comparison with the fault classification methods based on SVM and ELM, the experimental results show that the proposed method has higher classification accuracy and better generalization ability.
\end{abstract}

\section{Introduction}

As a key part of rolling mill, bearing operates in the environment of high temperature, high humidity, and heavy dust. Besides, bearing bears the largest impact force and load during production and it easily goes wrong under this circumstance. Thus, monitoring of bearing and timely classifying the faults into correct types are of great significance.

Recently, data-driven fault monitoring and classification methods have attracted more and more attention [1]. In fact, the diversity and quality of modeling data influence the effectiveness of the fault classification. Now, operating data of rolling mill are collected through wired communication, which needs high cost and are hard to be constructed. However, with the development of wireless communication technology, wireless sensor network (WSN) has been widely applied in industrial processes because it has the advantages of low power consumption, low cost, wireless communication, and so forth [2]. To the best of the authors' knowledge, the studies on fault classification of bearing based on WSN technology are rarely reported. Using the welldeveloped networking technologies, data transmissions and information exchanges within and between systems become more efficient, fast, and reliable $[3,4]$.

After collection of data, for fault classification of bearing, several crucial points should be discussed: (1) how to extract the fault features from the collected signal; (2) how to improve the classification accuracy of the fault identification model. Liu and Pan [5] extracted bearing fault feature in time domain based on the analysis of data characteristic. Similarly, Shuang and Meng [6] analyzed the vibration signal of rolling bearing by using principal component analysis (PCA) and extracted data element as the reflection of the main characteristics of fault case. However, the vibration signals usually contain a large number of nonlinear components, while PCA is not capable of copping with the nonlinear characteristics. The 
above-mentioned methods judge the status of the rolling bearings only from a view of single fault feature, which could not give a comprehensive presentation of fault case. For the multi-fault-features extraction, Malhi and Gao [7] proposed a method to construct a mixed domain feature set based on wavelet decomposition. However, the time series is only extracted by wavelet decomposition in the frequency domain and time domain features are not considered. On the contrary, Lei et al. [8] extracted features from frequency instead of time domain from six aspects, which include original vibration signal and its spectrum and the filtered and demodulated signal by wavelet packet. Qin et al. [9] developed a fault classification model based on an improved extreme learning machine (ELM) method. However, to better describe fault, more fault features are needed to measure the fault influences. That is, multiple fault features should be decomposed rather than one single fault vector.

As for the fault classification, intelligent algorithms have been gradually applied to bearing, such as artificial neural network [10] and least squares support vector machines [11]. The traditional back propagation neural network (BPNN) faces the problem of slow convergence rate, difficulty of configuring tunable parameters, and easily falling into the local optimum. In comparison with BPNN, the generalization performance of support vector machine (SVM) is improved. However, kernel function and its parameters are usually given according to human experiences. ELM [12] is a newly developed single hidden layer feed-forward neural network, which does not require continuously to adjust parameters of hidden layer. In ELM, iterative parameter optimization process of traditional neural network is replaced with solving linear equation groups and the outputs of the minimum norm least squares solution are employed as the weights of the network. Therefore, the network is trained one time without iterations. Compared with BPNN and SVM, ELM greatly improves the training speed and generalization ability, which has been successfully applied in the areas such as pattern recognition [13-18]. The idea of introducing ELM into fault classification for bearing is given in this paper. In fact, the adjustment of the weights of the input and hidden layer adopts random selection method, which cannot guarantee the validity of the weight. Because the structure parameters of fault identification model determine the classification ability, how to obtain the optimal structure parameters is the key to improve the classification accuracy of fault identification model.

To solve the above-mentioned problems, a comprehensive and effective fault feature extraction and classification algorithm is proposed in this paper. First, a WSN is constructed in gearbox to collect the vibration signal. Second, to overcome the influence of disturbances, morphological average filtering algorithm is given to filter the collected signals and then the intrinsic mode function (IMF) is obtained through empirical mode decomposition (EMD) [19] after denoising. IMF presents fault features and IMF components that have large correlation coefficients and are used to calculate energy index in time domain. Besides, on the other hand, envelope spectrums of these IMFs are obtained by Hilbert envelope spectrum analysis, which can be used to obtain the singular values by singular decomposition on envelope spectrum matrix. The singular matrix and the energy index reconstructed multiple feature vectors used for classification. Third, bat algorithm [20] is utilized to optimize the weights of input and hidden layers of ELM, which use dynamic control of global and local search to avoid the results falling into the local optimum. At last, the fault classification model is developed by these feature vectors and the optimized ELM algorithm. The major contribution of this article is summarized as follows:

(1) A data acquisition system based on wireless sensor network is constructed to replace the traditional wired system.

(2) A multiple fault features decomposition method is proposed to explain the fault influences using two indices with physical significance.

(3) A bat algorithm optimized ELM algorithm is proposed to determine the parameters to achieve better classification accuracy.

The remainder of this article is organized as follows. In Section 2, a simple description of rolling bearing and the developed WSN is briefly given. Next, the extraction of multiple feature vectors is proposed in Section 3. In Section 4, fault classification method is formulated based on the enhanced ELM algorithm. Experiment results clearly demonstrate the efficacy and feasibility of the proposed method in the last section.

\section{Description of Rolling Bearing and the Developed Wireless Sensor Network}

Rolling bearing has been widely used in industry, which is mainly composed of four parts: inner ring, outer ring, rolling body, and the holder. Figure 1 shows the physical structure of rolling bearing. Its main function is transforming sliding friction between the shaft and seat into rolling friction. The bearing studied in this paper is from Baotou Iron and Steel Group, of which the type is S-21062-C produced by the SOR Company, US.

To collect data from rolling bearing, wireless sensor network (WSN) is constructed. WSN usually consists of a number of sensor nodes, cluster head nodes, and sink nodes. Besides, it forms a multihop ad hoc network system through the wireless communication, which can be used to receive, send, and process the information of monitoring objects within the covered area [17]. Considering that the object is a low speed and heavy load mill, we design a WSN that is battery-powered and of low power consumption, low cost, and rapid deployment to monitor the vibration of rolling mill.

Figure 2 shows the developed network topology of CSP-F1 rolling mill gearbox, which consists of sensor nodes, cluster head, and sink nodes. The sensor node collects and then sends vibration signals to cluster head node according to the given sampling interval; meanwhile, it also receives commands from the cluster head nodes. Cluster head node collects measured vibration data from sensor nodes and transmits the data to sink node. Besides, cluster head node has three roles, which are sending command to cluster measured vibration 


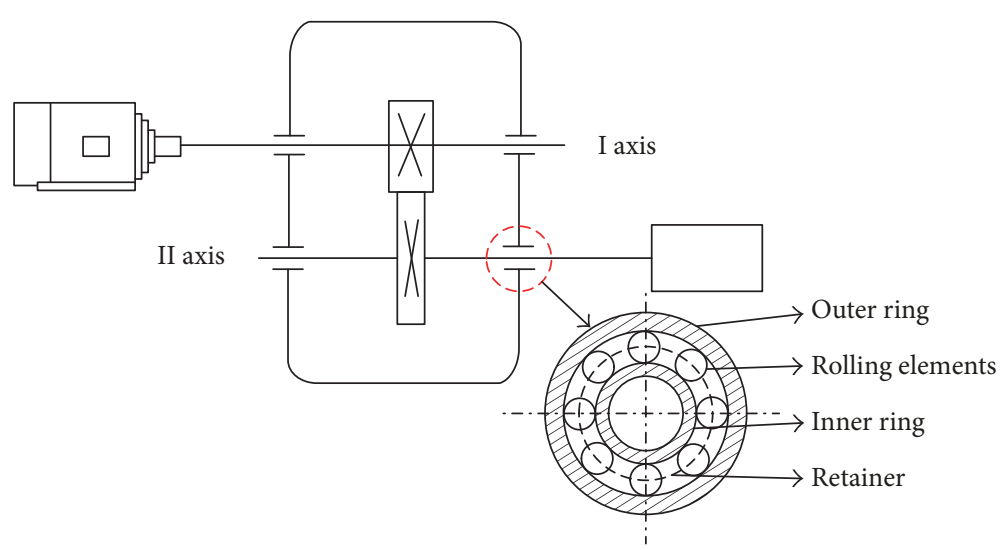

Figure 1: Physical structure of rolling bearing.

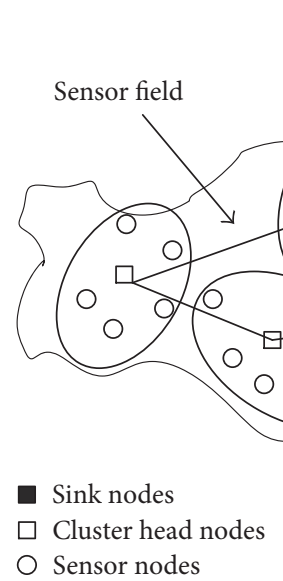

FIGURE 2: Network topology of wireless sensor network.

node, receiving the convergence order and maintenance time synchronization. The main functions of sink node are collecting data from cluster head node and transmitting the data to monitoring system.

\section{The Extraction of Multiple Feature Vectors}

In order to effectively extract plenty of information under different status of rolling bearing, the signals collected by WSN are denoised by morphological averaged filter. After denosing, Figure 3 shows the proposed procedure of feature extraction. Specifically, it includes three parts: (1) employing the EMD method to get intrinsic mode components (IMFs) that have large correlation coefficients; (2) calculating energy index based on the obtained IMFs; (3) performing Hilbert envelope analysis to IMFs to obtain the envelope spectrum and get their singular values. Through the above steps, the calculated energy index and singular values are employed as multiple feature vectors for bearing fault classification.

3.1. Denoising of Original Signals. To remove the noise contained in the data collected from WSN system, mathematical morphology (MM) and average filtering algorithm [18] are used for filtering. The idea of MM is to use some structural elements that have certain shapes to measure and extract images corresponding to the shape and achieve the purpose of image analysis. Based on the geometric characteristics of the signal, MM based average filter can cope with the nonlinear signal noise by morphological operations between structural elements and the original signal. The proposed filter inherits the advantages of $\mathrm{MM}$, including simple operation and analysis in time domain. Therefore, it is advantageous for the processing of mechanical fault signals.

Opening operator $\circ$ and closing operator $\bullet$ are two basic operations of MM, which are shown as follows, respectively:

$$
\begin{aligned}
& (f \circ g)(n)=(f \Theta g \oplus g)(n), \\
& (f \circ g)(n)=(f \oplus g \Theta g)(n),
\end{aligned}
$$

where $\Theta$ is erosion operation presenting the relationship in (3); $\oplus$ is dilation operation having the relationship in (4).

$$
\begin{aligned}
(f \Theta g)(n) & =\min [f(n+m)-g(m)], \\
(f \oplus g)(n) & =\max [f(n-m)+g(m)],
\end{aligned}
$$

where symbols $n$ and $m$ indicate sampling time satisfying $n$ larger than $m$.

The linear combination of (1) and (2) can be used to construct the average filter (AVG):

$$
\operatorname{AVG}(f)=\frac{(f \cdot g+f \circ g)}{2} .
$$

In this way, positive and negative impulses of the signal are eliminated. Besides, it can smooth the signal and reduce the signal noise.

\subsection{Extraction of Multiple Feature Vectors}

3.2.1. Calculation of IMF Components. Due to harsh operation conditions of rolling bearing, its vibration signal always contains process disturbances, including the resonance and external noise. Therefore, after denoising using (5), the EMD 


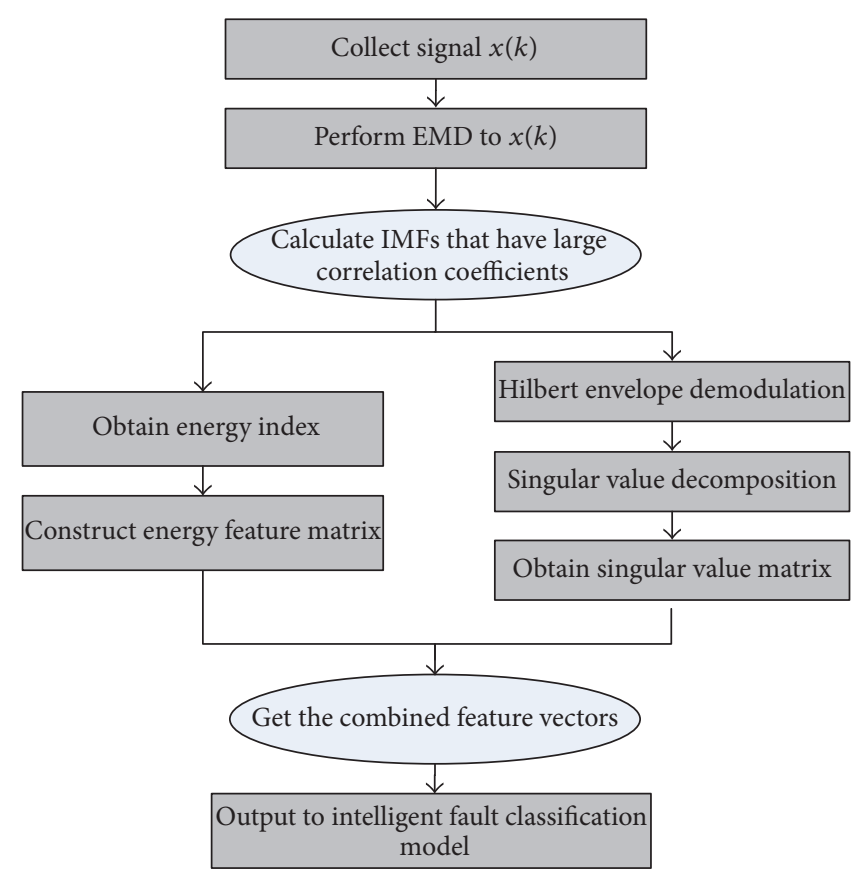

FIGURE 3: Flow chart of the extraction of fault feature vectors.

algorithm is employed to extract the inherent characteristics of signals. The concrete calculation procedures are given as follows.

Note the denoised signal still as $x(k)$ for brevity and decompose it according to EMD:

$$
x(k)=\sum_{i=1}^{m} c_{i}(k)+r_{m}(k),
$$

where $c_{i}(k) i \in[1, m]$ is IMF, $r_{m}(k)$ is the residual of the signal, and $k$ is the index of sampling time.

In fact, different IMFs have different significances in comparison with the original signal $x(k)$. And the significance can be evaluated by a correlation coefficient. Inspired by the definition of cross-correlation function, the correlation coefficient $\rho_{x c_{i}}$ between the original signal $x(k)$ and $\operatorname{IMF} c_{i}(k)$ is defined as follows:

$$
\rho_{x c_{i}}=\frac{\sum_{k=0}^{K} x(k) c_{i}(k)}{\sqrt{\sum_{k=0}^{K} x(k)^{2} \sum_{k=0}^{K} c_{i}(k)^{2}}},
$$

where $x(k)$ is the denoised signal, $c_{i}(k)$ is the $i$ th IMF, and $K$ is the number of sampling times.

A large value of the coefficient means that the corresponding IMF is relevant to the original signal. In this way, it eliminates the interference component and obtains the intrinsic component mode component that contains the most information of the original signal.

3.2.2. Energy Index. The values of $\rho_{x c_{i}}$ calculated from (7) are sorted in descending order. Then 0.1 is defined as the threshold of correlation coefficient and the first $m$ IMFs larger than the threshold are selected. On the basis of this, the energy index can be calculated as follows:

$$
E_{i}=\sum_{k=1}^{K} c_{i}(k) \quad i=1,2, \ldots, m .
$$

After that, an energy eigenvector $\mathbf{T}=\left[E_{1}, E_{2}, \ldots, E_{m}\right]$ is developed. For easy comparison and processing, $\mathbf{T}$ is normalized as follows:

$$
\mathbf{T}=\left[\frac{E_{1}}{E}, \frac{E_{2}}{E}, \ldots, \frac{E_{m}}{E}\right]^{T},
$$

where $E=\left(\sum_{i=1}^{m}\left|E_{i}\right|^{2}\right)^{1 / 2}$.

3.2.3. IMF Based Hilbert Envelope Spectrum Analysis. The IMFs $c_{1}, c_{2}, \ldots, c_{m}$ calculated from Section 3.2.2 are taken to perform Hilbert transform according to

$$
H\left[c_{i}(k)\right]=\frac{1}{\pi} \sum_{r=1}^{K} \frac{c_{i}(r)}{k-r} .
$$

Combined with (10), the envelope spectrum of each IMF is calculated as follows:

$$
B_{i}(k)=\sqrt{c_{i}^{2}(k)+H^{2}\left[c_{i}(k)\right]} .
$$

Finally, the envelope spectrum of each IMF constructs a matrix B. By performing the singular value decomposition theory [18] on $\mathbf{B}$, it obtains

$$
\mathbf{B}=\mathbf{U S V}^{T},
$$




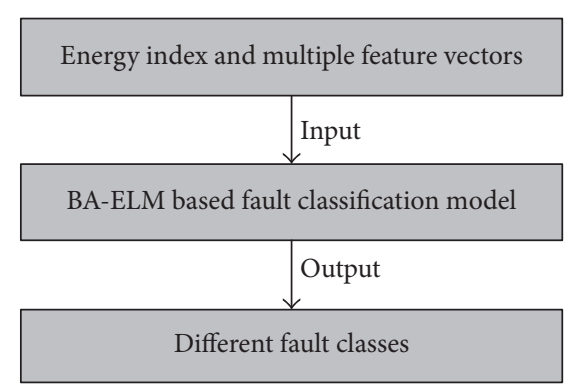

FIGURE 4: Flow chart of intelligent fault classification.

where $\mathbf{S}=\operatorname{diag}\left(\sigma_{1}, \sigma_{2}, \ldots, \sigma_{l}\right)$ is the singular values of the matrix $\mathbf{B} ; \mathbf{U}=\left[u_{1}, u_{2}, \ldots, u_{m}\right]$ and $\mathbf{V}=\left[v_{1}, v_{2}, \ldots, v_{n}\right]$ are orthogonal matrixes.

By processing each group of the signal under different status according to the above steps, we obtain the IMF Hilbert envelope spectrum singular value matrix and combine these singular value matrixes and energy features as multiple feature vectors to classify fault of rolling bearing. And multiple feature vectors are employed to train classification model of rolling bear based on bat algorithm (BA) optimized ELM, which will be given in the following section.

\section{Enhanced ELM Algorithm for Fault Classification}

The accuracy of fault classification depends on the intelligent model used in the process of machine learning methods. In comparison with the BP method and the SVM method, ELM only needs to determine the number of nodes of hidden layer during the training of the network. Besides, it has the advantages of high efficiency, fast learning speed, and the unique solution. However, two structure parameters of ELM, that is, input weights and hidden layer threshold, are randomly given, which may result in poor accuracy. Having the advantages of dynamic control of global and local search conversion and avoiding falling into local optimum, BA is employed to optimize the two structure parameters of ELM. Thus, BA optimized ELM is proposed in the developed rolling bearing fault classification model to improve the precision and generalization ability.

4.1. The Establishment of Fault Classification Model. In this part, the fault classification model is developed based on ELM. Figure 4 shows the proposed method. Only determining the number of neurons in hidden layer, ELM randomly generates connection weights and threshold of hidden layer neurons between the input layer and hidden layer and it can obtain the unique optimal solution.

Assuming that the number of samples is $N$, the number of nodes of hidden layer is $L$, and the activation function is $g(x)$, the mathematical model of ELM is defined as follows:

$$
y_{i}=\sum_{j=1}^{L} \beta_{j} g\left(w_{j} x_{i}+b_{j}\right)
$$

where $w_{j}=\left[k_{j 1}, k_{j 2}, \ldots, k_{j n}\right]$ is the connection weights vector between the input node and the $j$ th node of hidden layer; $b_{j}$ is threshold of the $j$ th node in hidden layer.

In (13), a feed-forward neural network model of single hidden layer is developed, of which the output is close to zero error:

$$
\sum_{i=1}^{N}\left\|y_{i}-t_{i}\right\|^{2}=0
$$

Sequentially, parameters $w_{j}, b_{j}$, and $\beta_{j}$ satisfy the following relationship:

$$
\sum_{i=1}^{N} \beta_{j} g\left(w_{j} x_{i}+b_{j}\right)=t_{i}, \quad i=1,2, \ldots, N .
$$

And (14) can be further simplified as $\mathbf{H} \boldsymbol{\beta}=\mathbf{T}$, in which

$$
\begin{aligned}
\mathbf{H} & =\left[\begin{array}{ccc}
g\left(k_{1}, b_{1}, x_{1}\right) & \ldots & g\left(k_{L}, b_{L}, x_{1}\right) \\
\ldots & \ldots & \ldots \\
g\left(k_{1}, b_{1}, x_{N}\right) & \ldots & g\left(k_{L}, b_{L}, x_{N}\right)
\end{array}\right]_{N \times L}, \\
\boldsymbol{\beta} & =\left[\begin{array}{c}
\beta_{1}^{T} \\
\vdots \\
\beta_{L}^{T}
\end{array}\right], \\
\mathbf{T} & =\left[\begin{array}{c}
T_{1}^{T} \\
\ldots \\
T_{N}^{T}
\end{array}\right]_{N \times m} .
\end{aligned}
$$

$\mathbf{H}$ is the output matrix of hidden layer and $\mathbf{H}(i, j)$ stands for the output of the $i$ th training data in the $j$ th hidden node.

The goal of adjustment is to find a set of optimal parameters $w_{j}, b_{j}, \beta_{j}$ that make the $\left\|(\mathbf{H} \boldsymbol{\beta})^{T}-\mathbf{T}\right\|$ minimum.

4.2. Enhanced ELM Based on BA. The weights of input layer and thresholds of hidden layer might be zero, which may result in the functionless of some hidden layers. Thus, the number of hidden layer nodes has to be increased to achieve higher classification accuracy. However, it may lead to poor adaptability and low generalization capacity for testing data. To solve this problem, BA is employed to optimize the input weights and threshold of hidden layer of ELM. In this way, the classification accuracy and generalization ability will be improved. Figure 5 shows the specific process.

BA is a new heuristic algorithm proposed by Yang et al. [21] and it has the advantages of fast convergence speed and high convergence precision. It is used to find the optimal solution of the problem by simulating the foraging behaviors of bat. The specifics are as follows:

(1) Initialize the bat population location $x_{i}^{t}$ and speed $v_{i}^{t}(i=1,2, \ldots, n)$, in which $t$ is the time index. Define the pulse frequency $f_{i}$ of the $i_{t}^{h}$ bats at position $x_{i}$. Then initialize the pulse firing rate $r_{i}^{t}$ and loudness $A_{i}^{t}$. According to the fitness value, determine the current optimal solution $x^{*}$. 


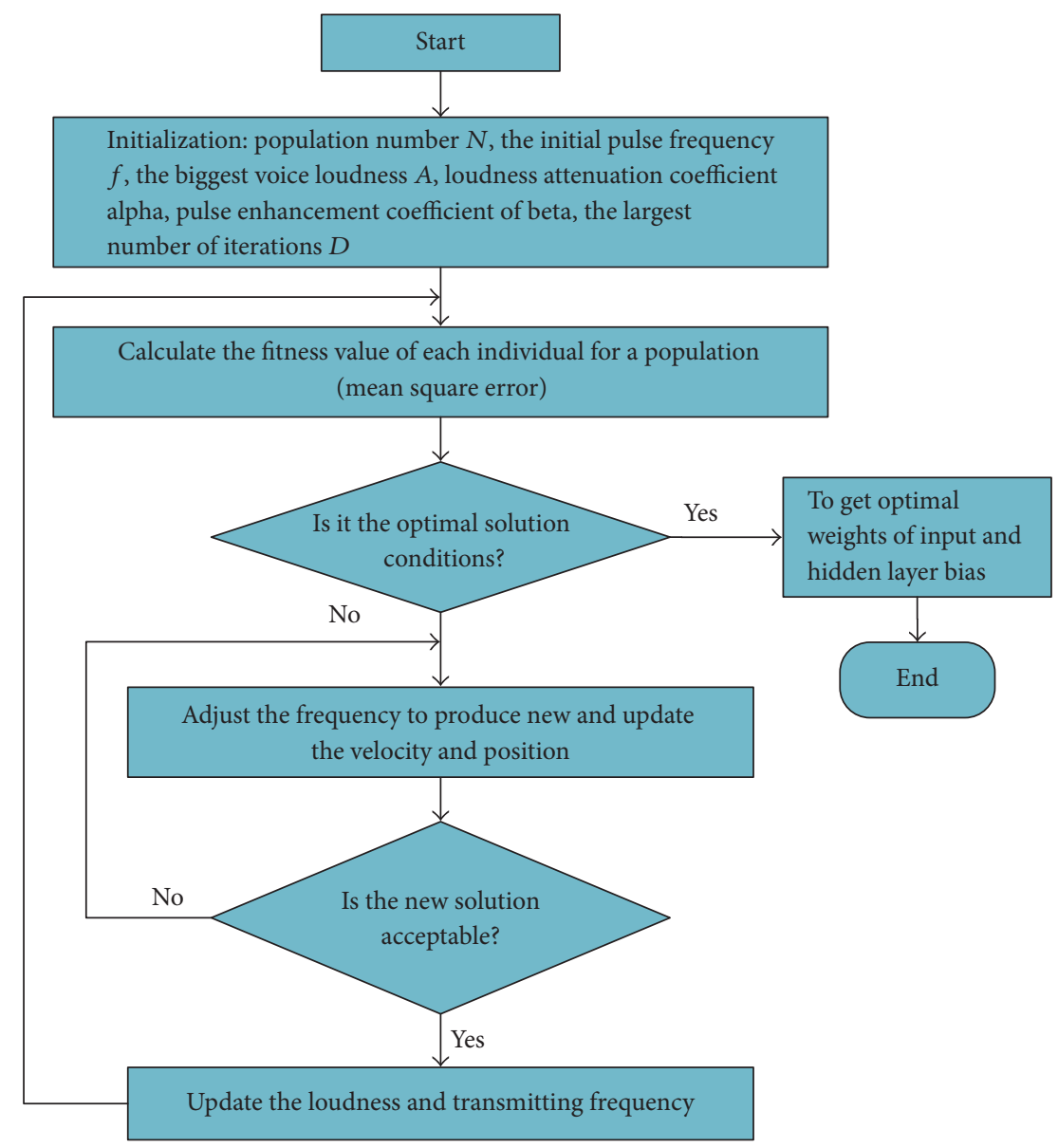

FIgURE 5: The flow chart of BA optimized ELM algorithm.

(2) Update the bat pulse frequency, speed, and position according to (17) through (19), respectively,

$$
\begin{aligned}
& f_{i}=f_{\min }+\beta\left(f_{\max }-f_{\min }\right), \\
& v_{i}^{t}=v_{i}^{t-1}+f_{i}\left(x_{i}^{t}-x^{*}\right), \\
& x_{i}^{t}=x_{i}^{t-1}+v_{i}^{t},
\end{aligned}
$$

where $\beta \in[0,1]$ is a random number uniformly distributed; $v_{i}^{t}, v_{i}^{t-1}$ are speed at time $t$ and $t_{1} ; x_{i}^{t}, x_{i}^{t-1}$ represent the position of the bat at times $t$ and $t_{1}$.

(3) Generate uniformly distributed random number $\rho_{1}$. If $\rho_{1}>r_{i}$, it means that a new solution is produced by random perturbations and then carry out crossborder for new solution.

(4) Generate uniformly distributed random number $\rho_{2}$. If $\rho_{2}>A_{i}$ and $f\left(x_{i}\right)<f\left(x^{*}\right)$, the solution of Step (3) is acceptable. Then update $r_{i}$ and $A_{i}$ according to

$$
\begin{aligned}
A_{i}^{t+1} & =\alpha A_{i}^{t}, \\
r_{i}^{t+1} & =R_{0}[1-\exp (-\gamma t)] .
\end{aligned}
$$

(5) Sort the fitness value of all bats and find out the optimal solution.

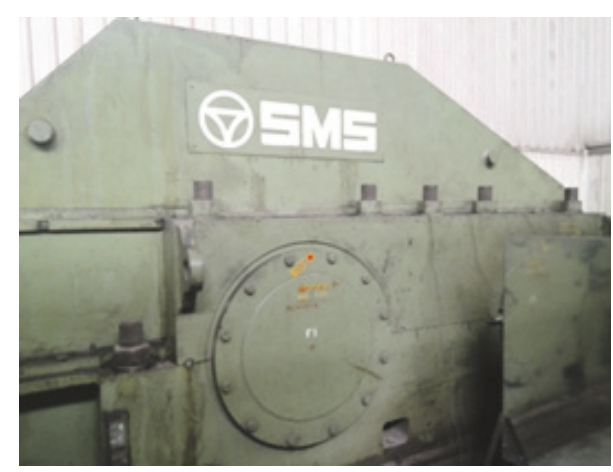

FIGURE 6: The gearbox of rolling mill.

(6) Repeat Steps (1)-(5) until a solution that meets the termination condition is found.

\section{Results and Discussions}

5.1. Data Preparations. The application object of this article is a mill located in Baotou Iron and Steel Group, China. Figure 6 is the gearbox of the mill, which is the source of power and its operation status greatly affects the whole production line. 


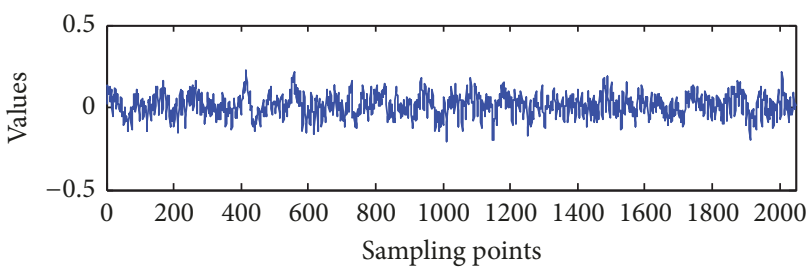

(a)

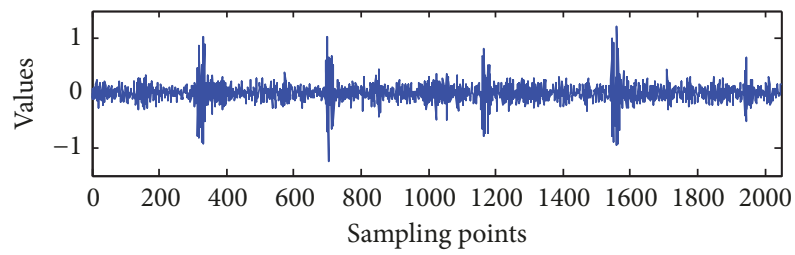

(c)

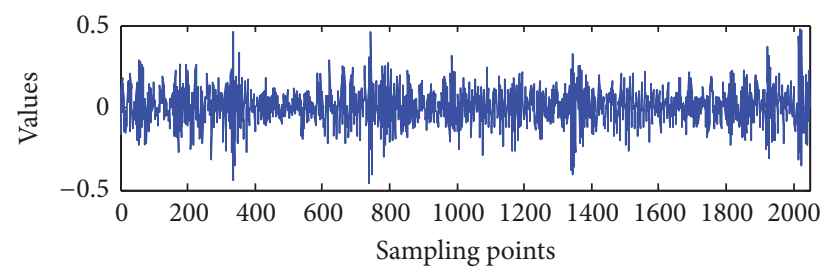

(b)

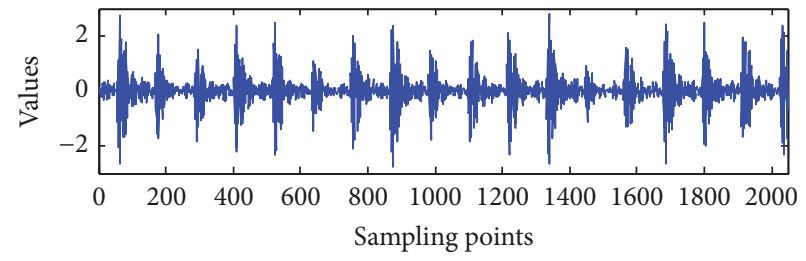

(d)

FigURE 7: Signal collected from WSN of (a) normal status, (b) rolling bearing fault, (c) inner ring fault, and (d) outer ring fault.

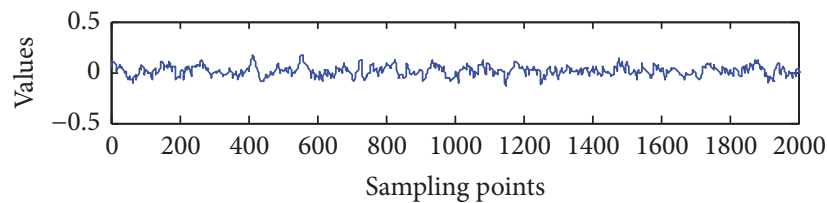

(a)

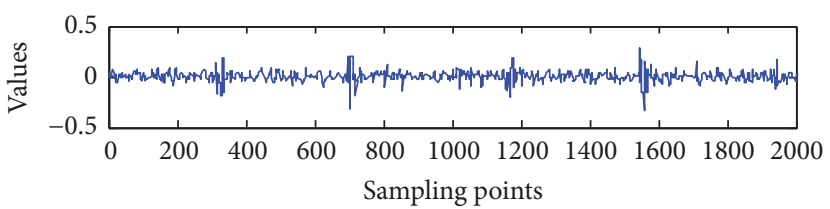

(c)

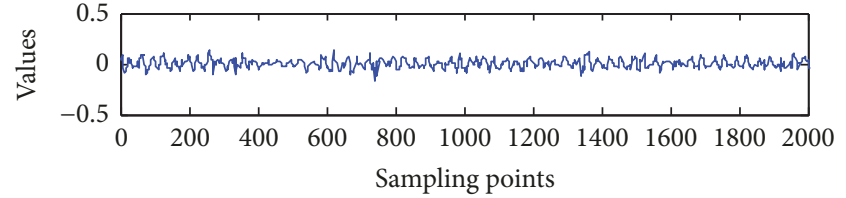

(b)

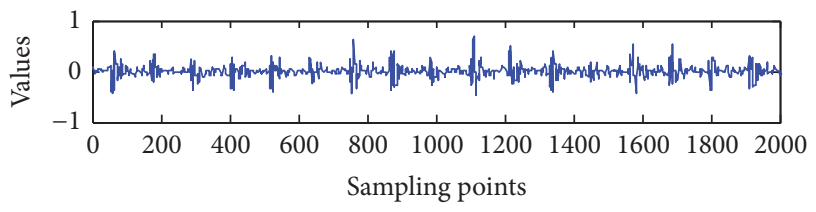

(d)

FIGURE 8: Waveforms after filtering (a) normal status, (b) rolling bearing fault, (c) inner ring fault, and (d) outer ring fault.

A data collection system based on WSN is constructed and vibration signal can be collected online. In common, there are three types of fault: rolling bearing fault, inner ring fault, and outer ring fault. Combined with the normal status, Figure 7 shows the four kinds of signal collected for analysis.

Morphological average filter is used to denoise the above signals. The linear structural element is selected, and each structural element value is 0 , namely, $g=\{0,0,0\}$. According to the determined structural elements, four states signals' noise is filtered by morphological average filter, as shown in Figure 8. In Figures 7(a) and 8(a), it can be observed that the noise of the normal signals is significantly reduced after morphological average filtering. The similar phenomena can be observed from other three fault cases.

For each operation status, experiment was performed 30 times. Each experiment contains 2048 data points. Then, EMD is used to decompose the state sample under different status. According to the rule given in Section 3.2.1, four IMFs will be retained. Figure 9 shows the decomposition of one experiment under normal status.

5.2. The Development of Classification Model. The correlation coefficient between the original signal and obtained IMF after

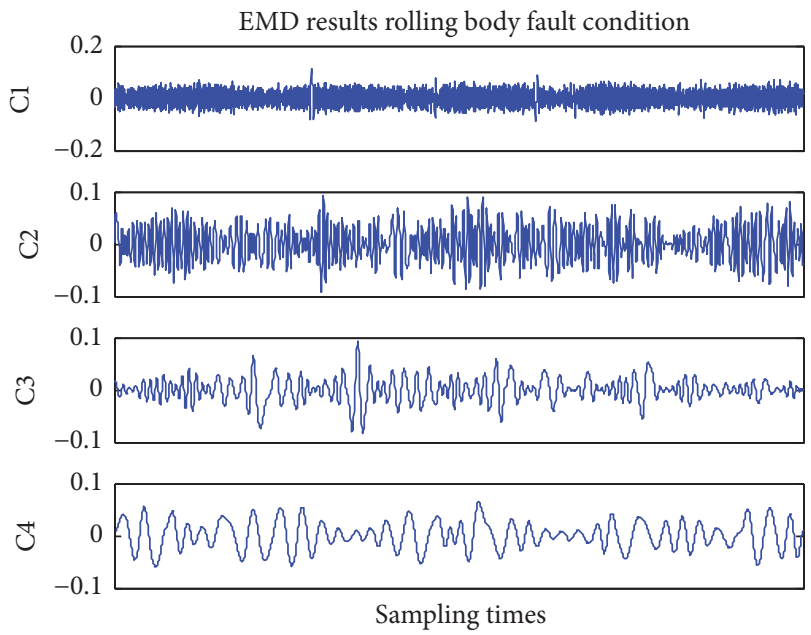

FIGURE 9: The results of EMD for normal condition.

decomposition of each state is evaluated. Table 1 summarized the results. Taking Hilbert envelope for these four IMFs, the results are shown in Figure 10. It is observed that approximate fault frequencies of different conditions are greatly different. 


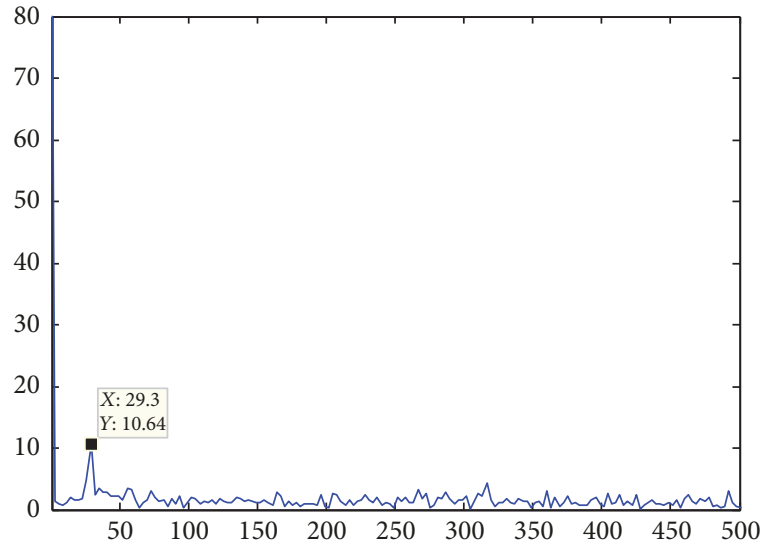

(a)

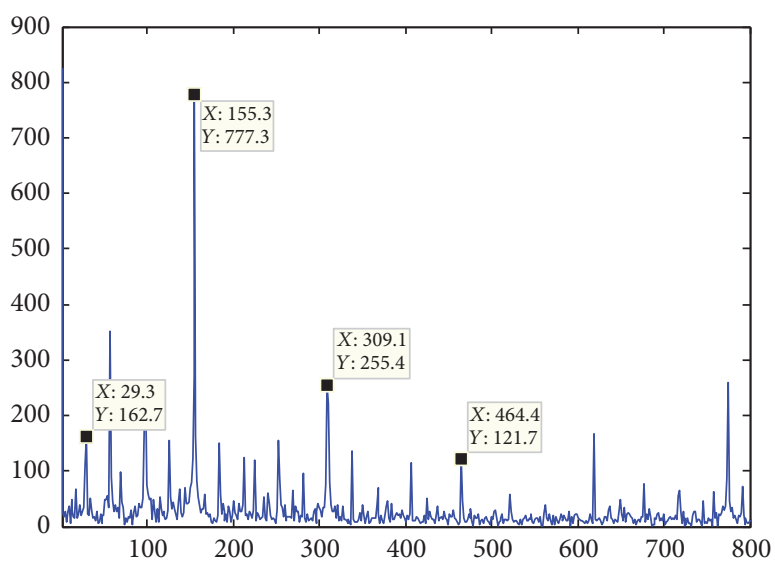

(c)

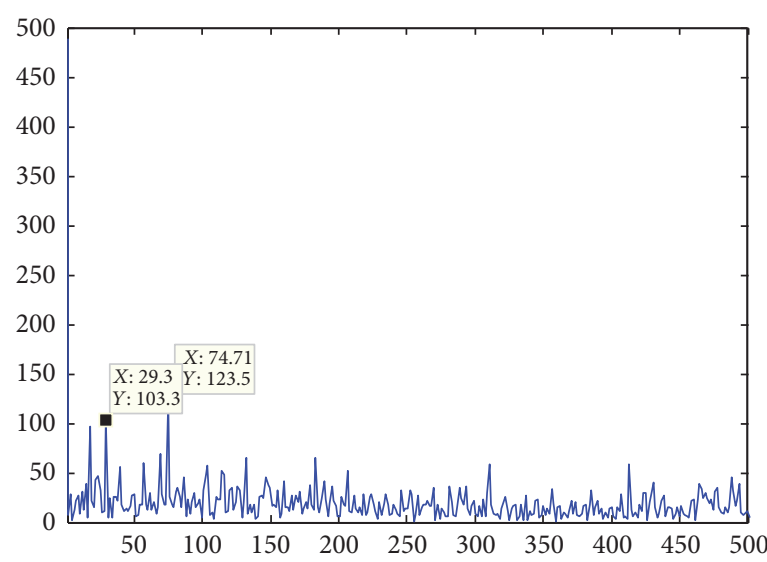

(b)

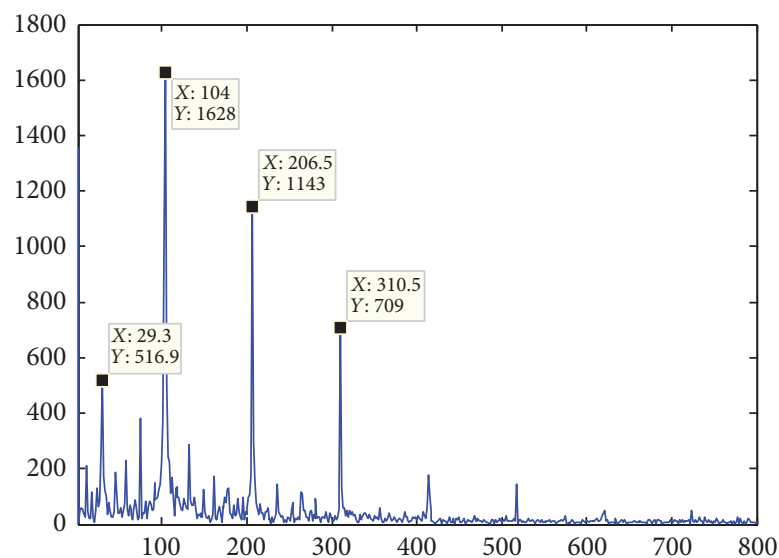

(d)

Figure 10: Hilbert envelope demodulation spectrum for (a) normal status, (b) rolling bearing fault, (c) inner ring fault, and (d) outer ring fault.

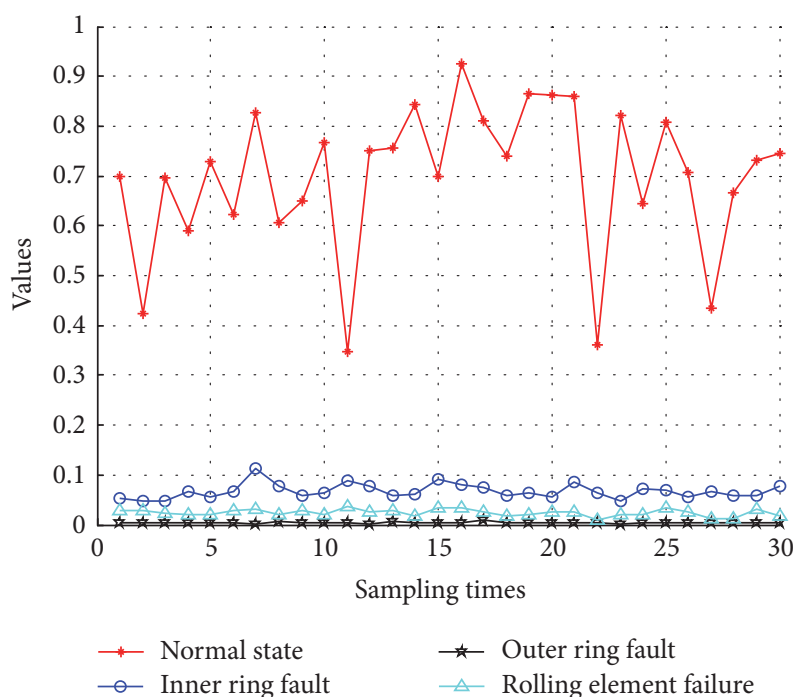

FIGURE 11: Time domain index (energy) for four cases.

Two indices, one from time domain and one from frequency domain, are calculated using the first four IMFs through the Hilbert envelope demodulation. Figures 11 and 12
TABLE 1: Correlation coefficients between IMFs and the original signal in four cases.

\begin{tabular}{lcccc}
\hline & $\mathrm{IMF}_{1}$ & $\mathrm{IMF}_{2}$ & $\mathrm{IMF}_{3}$ & $\mathrm{IMF}_{4}$ \\
\hline \multirow{3}{*}{ Normal } & 0.4452 & 0.5507 & 0.5020 & 0.2117 \\
& 0.0634 & 0.1692 & 0.3012 & 0.0501 \\
& 0.0419 & 0.1353 & 0.0801 & 0.0993 \\
\hline \multirow{3}{*}{ Fault 1 } & 0.8769 & 0.4236 & 0.1818 & 0.0940 \\
& 0.1529 & 0.0680 & 0.0355 & 0.0023 \\
& 0.0311 & 0.0598 & 0.0047 & 0.0110 \\
\hline \multirow{3}{*}{ Fault 2 } & 0.9929 & 0.0867 & 0.0115 & 0.0114 \\
& 0.0629 & 0.0045 & 0.0002 & 0.0042 \\
& 0.0446 & 0.0036 & 0.0015 & 0.0019 \\
\hline \multirow{3}{*}{ Fault 3 } & 0.9529 & 0.1038 & 0.1520 & 0.1546 \\
& 0.1569 & 0.0253 & 0.1300 & 0.0736 \\
& 0.0410 & 0.0206 & 0.0751 & 0.0352 \\
\hline
\end{tabular}

plot these two indices, respectively. The normal state has the highest energy value, followed with inner ring fault, and outer ring fault and the last one is rolling fault. However, outer ring fault presents the highest singular value of the Hilbert envelope and then is followed by the inner ring fault, rolling 


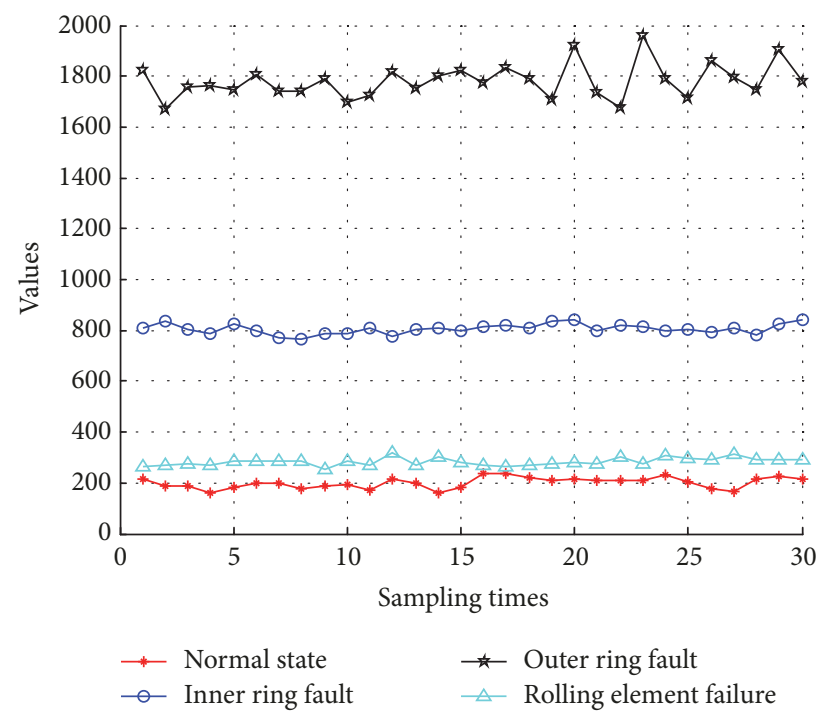

FIGURE 12: Frequency domain index (Hilbert envelope singular value) under four cases.

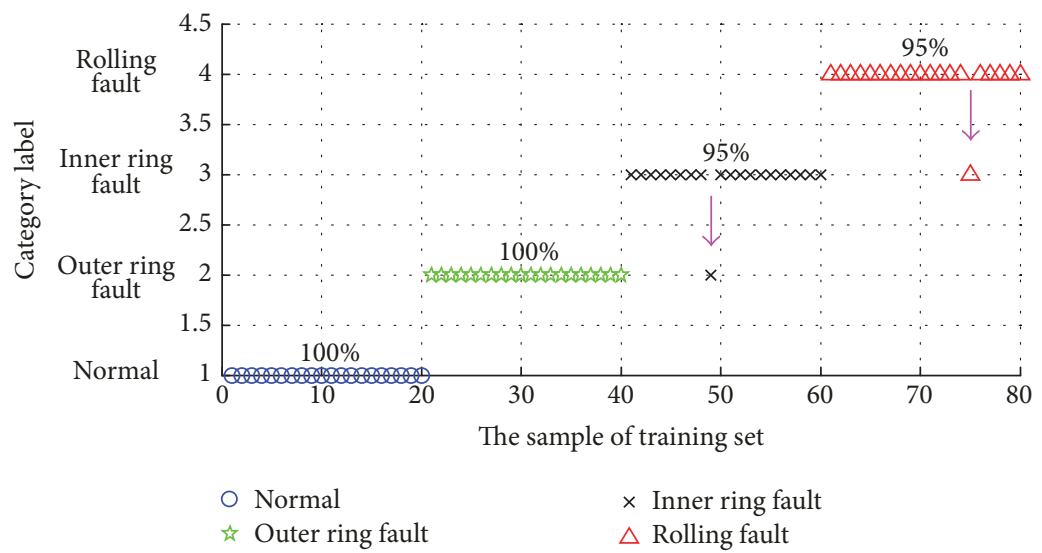

FIgURE 13: Classification of testing data based on BA-ELM.

bearing fault, and normal status. At the same time, it can be seen that under different conditions the discrimination ability of the two indices is very well and shows good performance.

For the proposed fault classification model, initial values of parameter of BA optimized ELM are as follows: the population number is 20 ; the range of pulse frequency is from $[0,2]$; the initial pulse frequency is 0.0001 ; the biggest voice loudness is 1.6; loudness attenuation coefficient is 0.9; pulse enhancement coefficient is 0.99; and the largest number of iterations is set to be 200. Totally, experiment data are repeated thirty times under each condition. Twenty of them are used as training data and the remaining ten are used as testing data. Using the energy index and Hilbert envelope spectrum singular value index as the input, the fault classification model based on the BA-ELM algorithm is developed. In Figure 13, fault classification accuracy of BA-ELM model for testing samples is $97.5 \%$, which is a high accuracy. The value of $y$-axis stands for the different operation status. If the value is 1 , it stands for normal condition. Similarly, inner ring fault, outer ring fault, and rolling bearing fault are
TABLE 2: Comparisons of SVM, ELM, and BA-ELM.

\begin{tabular}{lcccc}
\hline \multirow{2}{*}{ Algorithm } & \multicolumn{4}{c}{ Accuracy (\%) } \\
& Normal & Fault 1 & Fault 2 & Fault 3 \\
\hline SVM & 90 & 100 & 100 & 45 \\
ELM & 90 & 95 & 100 & 80 \\
BA-ELM & 100 & 100 & 95 & 95 \\
\hline
\end{tabular}

identified when the value is 2,3 , and 4 , respectively. To better illustrate the performance of the proposed method, SVM and the traditional ELM method are employed for comparison. Figure 14 shows the results of SVM and Figure 15 shows the results of ELM. Besides, these results are summarized in Table 2 for clear comparison. In summary, the proposed method has higher classification accuracy.

\section{Conclusion}

To solve the problems of data acquisition and fault classification for rolling bearing, several crucial points are solved in 


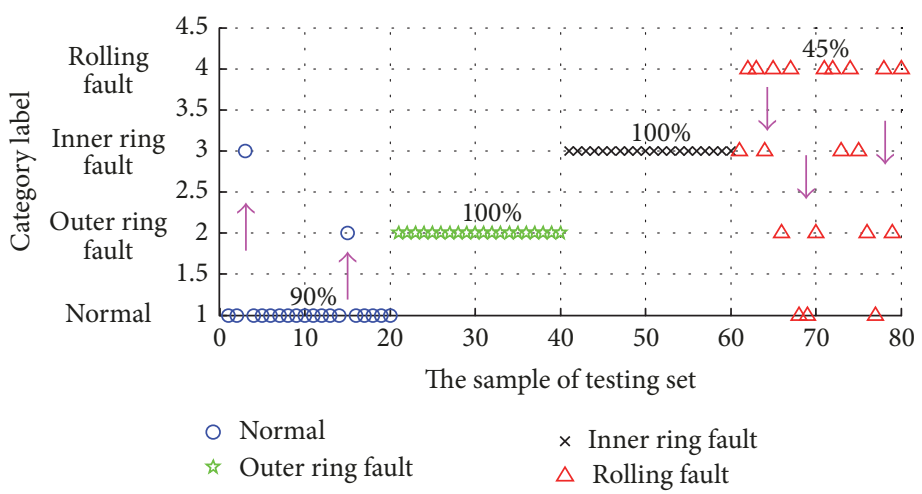

FIGURE 14: Classification of testing data based on SVM.

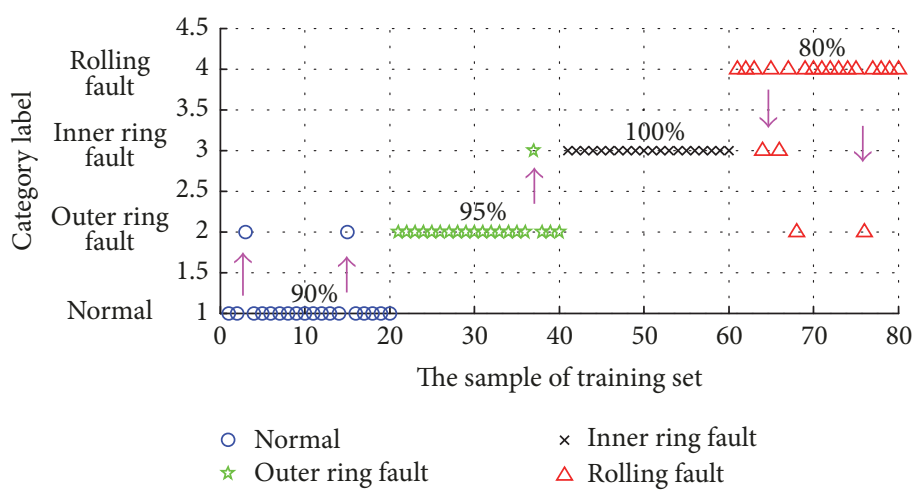

FIGURE 15: Classification of testing data based on ELM.

this paper. First, a data acquisition system based on wireless sensor network is constructed to replace the traditional wired system to collect sufficient data. Because rolling bearing works under a complex environment, the collected vibration signal is always polluted by noise. To effectively remove noise, a morphological average filtering algorithm is proposed. Then the empirical mode decomposition method is performed on the filtered data to obtain multiple feature vectors, including a frequency domain index and a time domain index. Then, these two indices are used as inputs for fault modeling. Finally, the fault classification model is developed based on enhanced extreme learning machine, which is optimized by bat algorithm to adjust the input weights and threshold of hidden layer node. In comparison with fault classification methods based on support vector machine and traditional extreme learning machine, the experimental results show that the proposed method has higher classification accuracy and better generalization ability.

\section{Conflicts of Interest}

The authors declare that they have no conflicts of interest.

\section{Acknowledgments}

This research is supported by the National Natural Science Foundation of China (no. 51565047), Natural Science Fund of Inner Mongolia (no. 2017MS0509), Innovation Fund of Inner Mongolia University of Science and Technology (no. 2015QDL12), and Innovation Fund of Inner Mongolia Postgraduate (no. S20171012708).

\section{References}

[1] Y. Qin, C. Zhao, and F. Gao, "An iterative two-step sequential phase partition (ITSPP) method for batch process modeling and online monitoring," AIChE Journal, vol. 62, no. 7, pp. 23582373, 2016.

[2] Y. Zhang, S. He, and J. Chen, "Data gathering optimization by dynamic sensing and routing in rechargeable sensor networks," IEEE/ACM Transactions on Networking, vol. 24, no. 3, pp. 16321646,2016

[3] Y. Hu, X. Xue, Z. Jin, and K. Peng, “Time-varying fault diagnosis for asynchronous multisensor systems based on augmented IMM and strong tracking filtering," Journal of Control Science and Engineering, Art. ID 5205698, 8 pages, 2018.

[4] H. Zhang, P. Cheng, L. Shi, and J. Chen, "Optimal denial-ofservice attack scheduling with energy constraint," Institute of Electrical and Electronics Engineers Transactions on Automatic Control, vol. 60, no. 11, pp. 3023-3028, 2015.

[5] R. Liu and F. Pan, "Roller Bearing Fault Diagnosis Based on SVM and BP neural network," Mechanical Engineering \& Automation, vol. 187, no. 6, pp. 32-134, 2014.

[6] L. Shuang and L. Meng, "Bearing fault diagnosis based on PCA and SVM," in Proceedings of the IEEE International Conference 
on Mechatronics and Automation (ICMA '07), pp. 3503-3507, Harbin, China, August 2007.

[7] A. Malhi and R. X. Gao, "PCA-based feature selection scheme for machine defect classification," IEEE Transactions on Instrumentation and Measurement, vol. 53, no. 6, pp. 1517-1525, 2004.

[8] Y. Lei, Z. He, and Y. Zi, "Application of an intelligent classification method to mechanical fault diagnosis," Expert Systems with Applications, vol. 36, no. 6, pp. 9941-9948, 2009.

[9] B. Qin, G. D. Sun, L. Y. Zhang, J. G. Wang, and J. Hu, "Fault Features Extraction and Identification based Rolling Bearing Fault Diagnosis," Journal of Physics: Conference Series, vol. 842, no. 1, Article ID 012055, 2017.

[10] N. Zheng, L. Zhang, W. Wang, B. Zhang, Y. Liu, and D. Zhang, "Research on fault diagnosis method based on rule base neural network," Journal of Control Science and Engineering, Article ID 8132528, 7 pages, 2017.

[11] J. Yang and J. Ma, "A sparsity-based training algorithm for Least Squares SVM," in Proceedings of the 5th IEEE Symposium on Computational Intelligence and Data Mining, CIDM 2014, pp. 345-350, USA, December 2014.

[12] G. B. Huang, Q. Y. Zhu, and C. K. Siew, "Extreme learning machine: theory and applications," Neurocomputing, vol. 70, no. 1-3, pp. 489-501, 2006.

[13] A. A. Mohammed, R. Minhas, Q. M. Jonathan Wu, and M. A. Sid-Ahmed, "Human face recognition based on multidimensional PCA and extreme learning machine," Pattern Recognition, vol. 44, no. 10-11, pp. 2588-2597, 2011.

[14] M. Van Heeswijk, Y. Miche, T. Lindh-Knuutila et al., "Adaptive ensemble models of extreme learning machines for time series prediction," Lecture Notes in Computer Science (including subseries Lecture Notes in Artificial Intelligence and Lecture Notes in Bioinformatics): Preface, vol. 5769, no. 2, pp. 305-314, 2009.

[15] H.-X. Tian and Z.-Z. Mao, "An ensemble ELM based on modified AdaBoost.RT algorithm for predicting the temperature of molten steel in ladle furnace," IEEE Transactions on Automation Science and Engineering, vol. 7, no. 1, pp. 73-80, 2010.

[16] Q. Yuan, W. Zhou, S. Li, and D. Cai, "Epileptic EEG classification based on extreme learning machine and nonlinear features," Epilepsy Research, vol. 96, no. 1-2, pp. 29-38, 2011.

[17] Z. Zhao, Z. Liu, Y. Sun, and J. Liu, "WOS-ELM-Based Double Redundancy Fault Diagnosis and Reconstruction for Aeroengine Sensor," Journal of Control Science and Engineering, vol. 2017, 14 pages, 2017.

[18] J. Wang, G. Xu, Q. Zhang, and L. Liang, "Application of improved morphological filter to the extraction of impulsive attenuation signals," Mechanical Systems and Signal Processing, vol. 23, no. 1, pp. 236-245, 2009.

[19] N. E. Huang, "Review of empirical mode decomposition," in Proceedings of the Wavelet Applications VIII, pp. 71-80, USA, April 2001.

[20] C. Rajeswari, B. Sathiyabhama, S. Devendiran, and K. Manivannan, "Diagnostics of gear faults using ensemble empirical mode decomposition, hybrid binary bat algorithm and machine learning algorithms," Journal of Vibroengineering, vol. 17, no. 3, pp. 1169-1187, 2015.

[21] X.-S. Yang and A. H. Gandomi, "Bat algorithm: A novel approach for global engineering optimization," Engineering Computations, vol. 29, no. 5, pp. 464-483, 2012. 


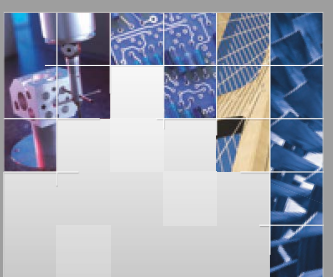

\section{Enfincering}
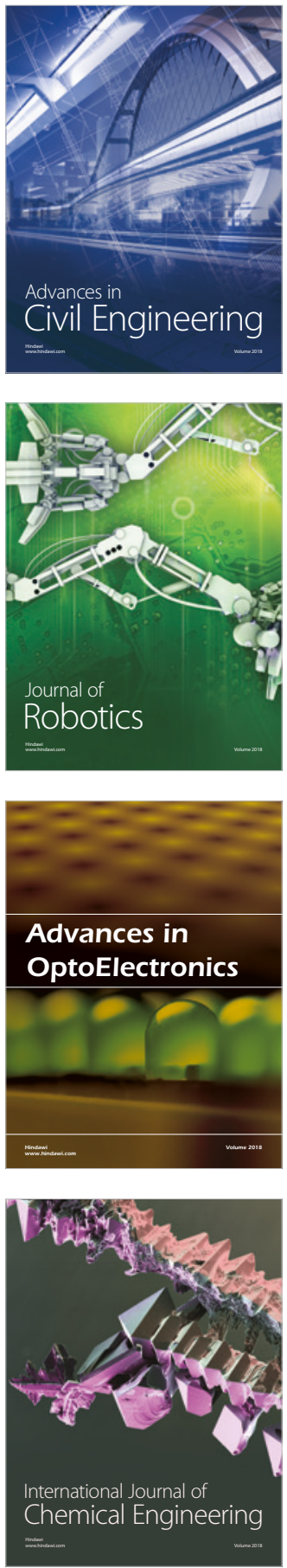

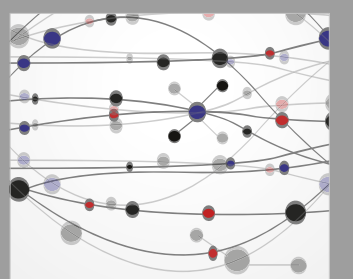

\section{Rotating \\ Machinery}

The Scientific World Journal

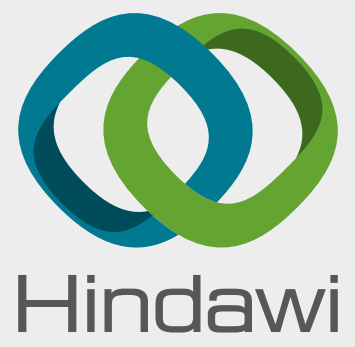

Submit your manuscripts at

www.hindawi.com
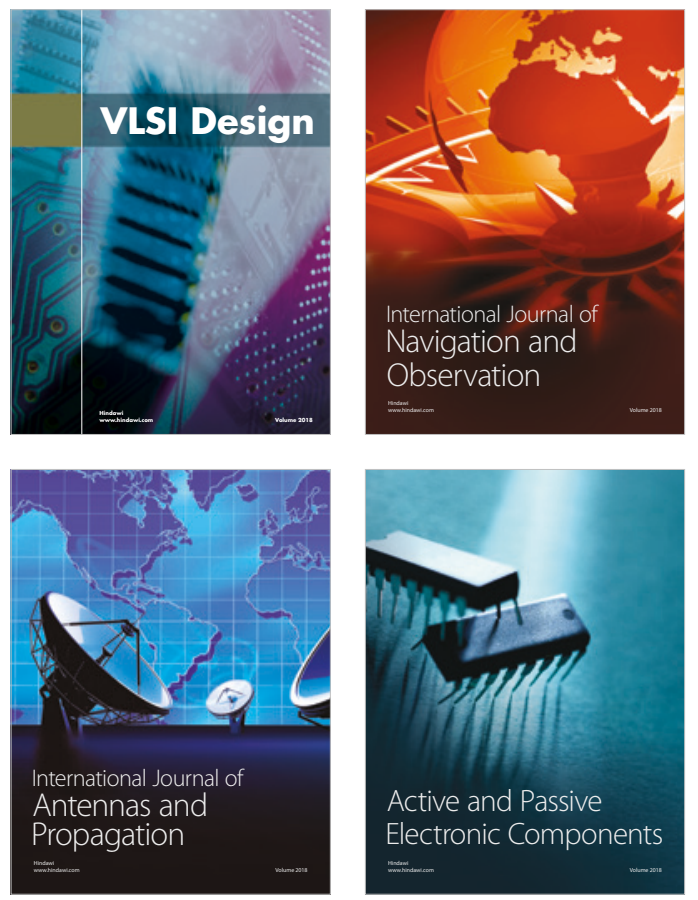
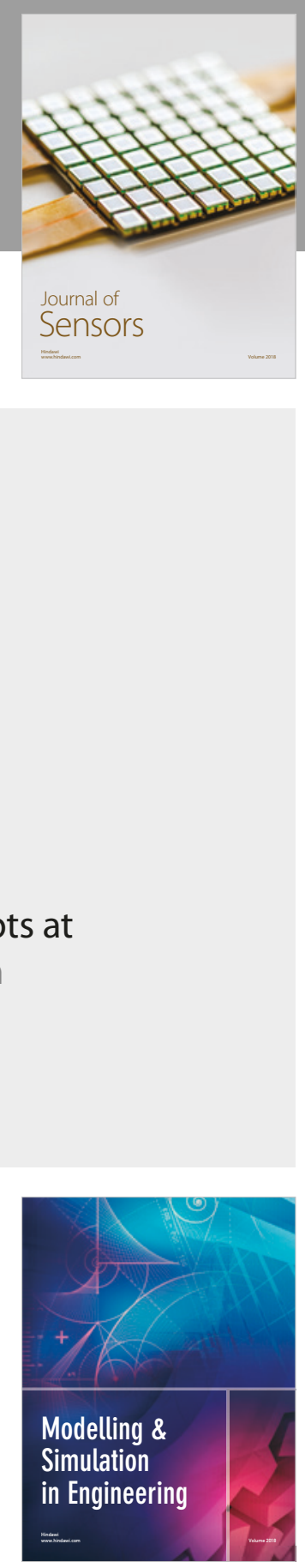

\section{Advances \\ Multimedia}
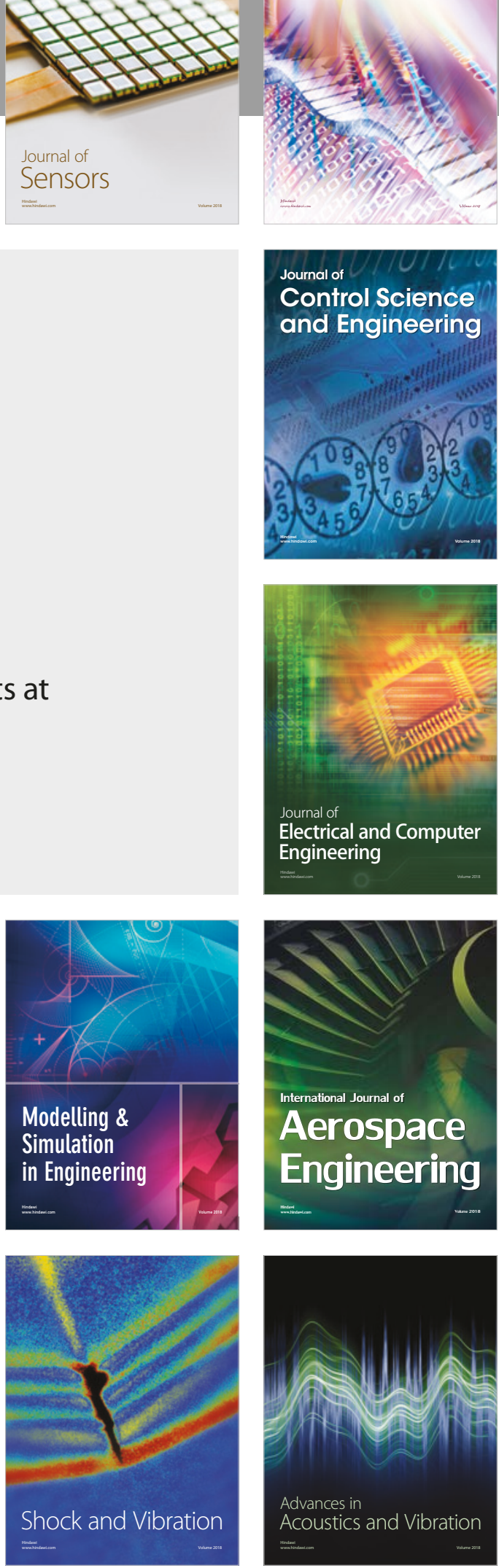